Pacific

Journal of

Mathematics

GROUPS THAT DO NOT ACT BY AUTOMORPHISMS OF CODIMENSION-ONE FOLIATIONS

R. Feres And D. Witte 


\title{
GROUPS THAT DO NOT ACT BY AUTOMORPHISMS OF CODIMENSION-ONE FOLIATIONS
}

\author{
R. Feres And D. Witte
}

\begin{abstract}
Let $\Gamma$ be a finitely generated group having the property that any action of any finite-index subgroup of $\Gamma$ by homeomorphisms of the circle must have a finite orbit. (By a theorem of É. Ghys, lattices in simple Lie groups of real rank at least 2 have this property.) Suppose that such a $\Gamma$ acts on a compact manifold $M$ by automorphisms of a codimension-one $C^{2}$ foliation, $\mathcal{F}$. We show that if $\mathcal{F}$ has a compact leaf, then some finite-index subgroup of $\Gamma$ fixes a compact leaf of $\mathcal{F}$. Furthermore, we give sufficient conditions for some finite-index subgroup of $\Gamma$ to fix each leaf of $\mathcal{F}$.
\end{abstract}

\section{Introduction and statement of results.}

The letter $M$ will denote a compact, connected, boundaryless, smooth manifold of dimension $n$. Let $\mathcal{F}$ be a $C^{r}$ foliation of $M$ by smooth leaves, $r \geq 2$. It will be assumed that $\mathcal{F}$ is a transversely oriented, codimension-one foliation.

Let $\mathcal{D}^{s}(M, \mathcal{F})$ denote the group of $C^{s}$ automorphisms of $\mathcal{F}, s \geq 0$; that is, the group of $C^{s}$ diffeomorphisms of $M$ that map leaves to leaves. The normal subgroup consisting of automorphisms of $\mathcal{F}$ that send each leaf to itself will be denoted $\mathcal{D}^{s}(M, \mathcal{F})_{0}$, and will be called the group of inner automorphisms of the foliation. The quotient

$$
\mathcal{O}^{s}(M, \mathcal{F})=\mathcal{D}^{s}(M, \mathcal{F}) / \mathcal{D}^{s}(M, \mathcal{F})_{0}
$$

will be called the group of transverse automorphisms (or outer automorphisms) of $\mathcal{F}$. When a group $\Gamma$ acts by automorphisms of $\mathcal{F}$ so as to define a homomorphism into $\mathcal{D}^{s}(M, \mathcal{F})$, the action will be called transversely finite if $\Gamma$ projects to a finite subgroup of $\mathcal{O}^{s}(M, \mathcal{F})$.

The general question that motivates the results of the present paper can be stated thus: Given $(M, \mathcal{F})$, what groups can act by automorphisms of $\mathcal{F}$ so that the action is not transversely finite, and what groups cannot? For example, if $\mathcal{F}$ is the foliation by fibers of a product manifold $M=B \times L$, with leaves $\{b\} \times L, b \in B$, then any group that acts nonfinitely on $B$ by $C^{s}$ diffeomorphisms also acts nonfinitely by outer-automorphisms of $\mathcal{F}$ (e.g., by setting the action on $L$ to be trivial). In this case, $\mathcal{O}^{s}(M, \mathcal{F})$ is the group of $C^{s}$-diffeomorphisms of $B$. If, on the other hand, $M=\mathbb{T}^{n}$ (the $n$-dimensional 
flat torus) and $\mathcal{F}$ is the foliation by planes parallel to an irrational hyperplane $F \subset \mathbb{R}^{n}$, then it is an elementary fact that $\mathcal{O}^{s}(M, \mathcal{F})$ is isomorphic to $H:=\left(\Gamma \ltimes \mathbb{R}^{n}\right) /\left(\Gamma_{0} \ltimes F\right)$, where $\Gamma$ is the stabilizer of $F$ in $G L(n, \mathbb{Z})$ and $\Gamma_{0}$ is the subgroup of $\Gamma$ that acts trivially on the quotient $\mathbb{R}^{n} / F$. In this case, only groups that admit homomorphisms with nonfinite image in $H$ can have nonfinite actions by outer-automorphisms of $\mathcal{F}$. (Allowing big codimension, it is quite easy to construct, say, topologically transitive foliated bundles, with large groups of smooth outer-automorphisms.)

A more specific question that will be addressed here is the following: Suppose that no (topological, say) action of a group $\Gamma$ on the circle yields nontrivial dynamics (that is, nonfinite action). Does $\Gamma$ admit nontrivial "transverse dynamics" on some codimension-one foliation of a compact manifold? The two theorems given below provide support for the negative answer.

Theorem 1.1. Suppose that $\Gamma$ is a finitely generated discrete group such that every homomorphism from a finite-index subgroup of $\Gamma$ into the group of homeomorphisms of the circle has a finite orbit on the circle. Also suppose that $\Gamma$ acts by $C^{0}$-automorphisms of $(M, \mathcal{F})$.

If $\mathcal{F}$ has a closed leaf, then some closed leaf of $\mathcal{F}$ is fixed by some subgroup of finite index in $\Gamma$.

By a bounded transverse invariant measure we mean a holonomy invariant transverse measure that is finite on compact transversals.

Corollary 1.2. Suppose that $\Gamma$ is a finitely generated discrete group such that every homomorphism from a finite-index subgroup of $\Gamma$ into the group of homeomorphisms of the circle has a finite orbit on the circle. Also suppose that $\Gamma$ acts by $C^{0}$-automorphisms of $(M, \mathcal{F})$.

If $\mathcal{F}$ admits a bounded transverse invariant measure, then it also admits a bounded transverse invariant measure $\mu$ that is invariant under $\Gamma$, such that every leaf in the support of $\mu$ is sent to itself under the action of a finite-index subgroup $\Gamma^{\prime}$ of $\Gamma$.

The next theorem provides a class of foliations on which the $\Gamma$-action is transversely finite. A foliation is said to be almost without holonomy if the germinal holonomy groups of all the non-compact leaves are trivial [7, IV2.11, p. 251]. This is the case, for example, if the non-compact leaves are simply connected.

Theorem 1.3. Suppose that $\Gamma$ is a finitely generated discrete group such that every homomorphism from a finite-index subgroup of $\Gamma$ into the group of homeomorphisms of the circle has a finite orbit on the circle.

If $\mathcal{F}$ is almost without holonomy, then every homomorphism of $\Gamma$ into $\mathcal{D}^{1}(M, \mathcal{F})$ yields a transversely finite action.

It is rather likely that the assumption about the holonomy of $\mathcal{F}$ in Theorem 1.3 can be greatly relaxed. 
The following theorem of É. Ghys [6] provides examples of groups that satisfy the requirements of Theorems 1.1 and 1.3, that is, groups for which every homomorphism into the group of homeomorphisms of the circle has a finite orbit. Most of these examples were also established by M. Burger and N. Monod $[\mathbf{1}, \mathbf{2}]$.

Theorem 1.4 (Ghys [6]). Suppose that $\Gamma$ is an irreducible lattice in a connected, semisimple, real Lie group $G$ of real rank at least 2, and that there is no continuous homomorphism from $G$ onto $P S L(2, \mathbb{R})$. Then every homomorphism from $\Gamma$ into the group of homeomorphisms of the circle, $\mathbb{T}^{1}$, has a finite orbit. Furthermore, if $\Gamma$ acts by $C^{1}$ diffeomorphisms, then some finite-index subgroup of $\Gamma$ acts trivially on $\mathbb{T}^{1}$.

\section{General facts about codimension-one foliations.}

We use [3] as our main reference for the fundamental concepts and results about codimension-one foliations. These results were established by R. Sacksteder, P. Dippolito, G. Hector, A. Haefliger, J. Cantwell, L. Conlon, and others. Some of the original sources are $[\mathbf{5}, \mathbf{9}, \mathbf{1 2}, \mathbf{1 4}, \mathbf{4}]$.

The foliation $\mathcal{F}$ will be said to be without holonomy if the germinal holonomy group of each leaf of $\mathcal{F}$ is trivial.

Let $\mathcal{L}$ denote a smooth one-dimensional foliation of $M$ everywhere transverse to $\mathcal{F}$. (Cf. $[3,5.1 .2]$.) It is convenient to work with biregular coordinate charts for the pair $(\mathcal{F}, \mathcal{L})$. These are charts that define foliation boxes for both $\mathcal{L}$ and $\mathcal{F}$ simultaneously, having local coordinate maps $\varphi: U \subset M \rightarrow V \subset \mathbb{R}^{n-1} \times \mathbb{R}^{1}, \varphi(p)=(x(p), y(p))$, such that $x=$ constant corresponds to plaques of $\mathcal{L}$ while $y=$ constant corresponds to plaques of $\mathcal{F}$. A biregular cover is an atlas comprised of biregular coordinate charts. Such covers exist. (Cf. $[3,5.1 .4]$.) From now on $\mathcal{L}$ will denote a fixed transverse foliation to $\mathcal{F}$ and any foliation box will be assumed without mention to be biregular.

An open $\mathcal{F}$-saturated set $U$ is a called a foliated product if it is connected and $\left.\mathcal{L}\right|_{U}$ fibers $U$ by open intervals over some $(n-1)$-dimensional manifold $B$. Since $\mathcal{F}$ is orientable, a foliated product is a trivial interval bundle, homeomorphic to $B \times(0,1)$ (although the foliation need not be the product foliation). Each leaf of $\mathcal{F}$ in $U$ with the restriction to it of the bundle map is a covering space of $B$. We note, in particular, that each closed transversal that meets $U$ has to meet every leaf in $U$. Let $d$ be the topological metric on $U$ induced by the restriction to $U$ of a Riemannian metric on $M$ and denote by $\widehat{U}$ the completion of $U$ in the metric $d$.

An $\mathcal{F}$-saturated set $U$ is a called a foliated bundle if it is connected and $\left.\mathcal{L}\right|_{U}$ fibers $U$ over some $(n-1)$-dimensional manifold $B$. (This is more general than a foliated product, because there is no restriction on the fibers.) An $\mathcal{F}$ saturated set $U$ is a called a trivially foliated product if there is a connected 
1-manifold $F$ (possibly with boundary), a connected $(n-1)$-dimensional manifold $B$, and a diffeomorphism from $U$ to $B \times F$, that carries $\left.\mathcal{L}\right|_{U}$ and $\left.\mathcal{F}\right|_{U}$ to the product foliations of $B \times F$.

Theorem 2.1 (Dippolito [5]). Let $U$ be a connected $\mathcal{F}$-saturated open set. Then $\widehat{U}$ is a connected manifold with finitely many boundary components. The interior of $\widehat{U}$ is $U$ and the inclusion $i: U \rightarrow M$ extends to an immersion $\hat{\imath}$ of $\widehat{U}$ into $M$ that sends the boundary components of $\widehat{U}$ onto boundary leaves of $U$. If $L^{\prime}$ is a boundary leaf of $U$ then $\hat{\iota}^{-1}\left(L^{\prime}\right)$ consists of one or two components of the boundary of $\widehat{U}$, each component being mapped bijectively to $L^{\prime}$ by $\hat{\imath}$. Both $\mathcal{F}$ and $\mathcal{L}$ pull-back under $\hat{\iota}$ to well-defined foliations on $\widehat{U}$. If $U$ is a foliated product, then $\widehat{U}$ is a foliated bundle whose fibers are compact intervals.

Proof. This is $[\mathbf{3}, 5.2 .10,5.2 .11,5.2 .12]$ as well as the remarks after 5.2.12 of the same reference.

The foliation of $\widehat{U}$ obtained by the pull-back of $\mathcal{F}$ will be denoted $\widehat{\mathcal{F}}$.

Theorem 2.2 (Sacksteder [14]). Let $\mathcal{F}$ be a transversely orientable foliation of class $C^{2}$ and codimension one on a compact manifold. Then the following are equivalent:

1) There exists a bounded transverse invariant measure $\mu$.

2) Either $\mathcal{F}$ has a compact leaf or $\mathcal{F}$ is without holonomy.

Proof. This is $[\mathbf{1 0}, 2.3 .8]$.

A foliation $\mathcal{F}$ of a manifold $U$ will be said to fiber over a manifold $B$ if there is a fibration of $U$ with base $B$ having the leaves of $\mathcal{F}$ as fibers.

Theorem 2.3. Let $\mathcal{F}$ be a transversely orientable foliation of class $C^{2}$ and codimension-one of a compact manifold $M$. Let $U$ be a connected $\mathcal{F}$-saturated open set and suppose that $\left.\mathcal{F}\right|_{U}$ is without holonomy. Then either every leaf of $\left.\mathcal{F}\right|_{U}$ is closed and $\left.\mathcal{F}\right|_{U}$ fibers over a connected 1-manifold, or each leaf of $\left.\mathcal{F}\right|_{U}$ is dense in $U$. Furthermore, if $\left.\mathcal{F}\right|_{U}$ is a fibration over a 1-manifold $B$, but $(\widehat{U}, \widehat{\mathcal{F}})$ is not a trivially foliated product, then $B \cong S^{1}$.

Proof. This is $[\mathbf{3}, 9.1 .4,9.1 .6]$.

Theorem 2.4. Let $\mathcal{F}$ be a transversely orientable foliation of class $C^{2}$ and codimension-one. Let $U \subset M$ be a connected, nonempty, open, $\mathcal{F}$-saturated set.

1) Suppose that $\left(U,\left.\mathcal{F}\right|_{U}\right)$ is without holonomy. Then there is a $C^{0}$ flow

$$
\Phi: \mathbb{R} \times \widehat{U} \rightarrow \widehat{U}
$$

that fixes the points of $\partial \widehat{U}$, carries leaves diffeomorphically to leaves and is transitive on the set of leaves of $\left.\mathcal{F}\right|_{U}$. Furthermore, $\left.\mathcal{F}\right|_{U}$ admits 
a bounded transverse-invariant nonatomic measure $\mu$ of full support that assigns to each transverse arc $\left\{\Phi_{t}(p) \mid t \in(a, b)\right\}$ the measure $b-a$.

2) Conversely, if $\left(U,\left.\mathcal{F}\right|_{U}\right)$ admits a bounded nonatomic transverse invariant measure $\mu$ of full support, then $\left.\mathcal{F}\right|_{U}$ is without holonomy and there exists a continuous flow $\Phi$ that carries leaves diffeomorphically to leaves, transitively on the set of leaves in $\left.\mathcal{F}\right|_{U}$, and fixing the leaves on the boundary. The flow $\Phi$ is related to $\mu$ in the way described in Part 1) of this theorem.

Proof. This is a special case of $[\mathbf{3}, 9.2 .1]$. The transverse-invariant measure is described in the proof given in the reference. See also [12].

Finally, we mention the following basic fact. For simplicity, we state it for a boundaryless $M$ and a $C^{1}$ foliation, although these conditions can be weakened. (Cf. [3, 6.1.1].)

Theorem 2.5 (Haefliger [8]). Let $\mathcal{F}$ be a codimension-one $C^{1}$ foliation of a compact connected boundaryless manifold $M$. Then the union of the compact leaves of $\mathcal{F}$ is a compact subset of $M$.

\section{Proof of Theorem 1.1.}

Suppose that a $\Gamma$-action satisfying the assumptions of Theorem 1.1 has been fixed. We may assume, by passing to a finite-index subgroup of $\Gamma$, that the $\Gamma$-action preserves the transverse orientation for $\mathcal{F}$.

The $\Gamma$-action will be said to be fixing if there exists a finite-index subgroup $\Gamma^{\prime}$ of $\Gamma$ and a compact leaf $L$ such that every element of $\Gamma^{\prime}$ sends $L$ to itself.

A nonempty $\mathcal{F}$-saturated subset $\mathcal{P}$ of $M$ will be called $\mathcal{F}$-perfect if for every differentiable curve $\alpha:(-a, a) \rightarrow M, a>0$, transverse to $\mathcal{F}$, the intersection of $\mathcal{P}$ with the image of $\alpha$ is a perfect set.

Lemma 3.1. If $\mathcal{F}$ has a compact leaf and the $\Gamma$-action is not fixing, then there exists a compact, $\mathcal{F}$-saturated, $\mathcal{F}$-perfect, $\Gamma$-invariant set $\mathcal{P} \subset M$, that is the union of compact, mutually homeomorphic leaves.

Proof. Let $\mathcal{C}$ denote the union of all compact leaves of $\mathcal{F}$ of a same homeomorphism type. A nonempty set of this kind exists since $\mathcal{F}$ has a compact leaf. It is also clear that $\mathcal{C}$ is invariant under every automorphism of $\mathcal{F}$. Furthermore, by a theorem of Haefliger, $[3,6.1 .1], \mathcal{C}$ is compact. Let

$\mathcal{A}=\{A \subset \mathcal{C} \mid A$ is compact, nonempty, $\mathcal{F}$-saturated, and $\Gamma$-invariant $\}$.

By Zorn's lemma, $\mathcal{A}$ has an element $A$ that is minimal under inclusion.

We define the derived set $A^{\prime}$ of $A$ as the subset of $A$ comprised of the union of all leaves $L \subset A$ whose points are limits of sequences in the complement of $L$ in $A$. If $A$ is the union of finitely many leaves, a finite-index subgroup 
of $\Gamma$ would send each of those finitely many leaves to itself, contradicting the assumption that the $\Gamma$-action is not fixing. Therefore $A^{\prime}$ is nonempty. It is easy to see that $A^{\prime} \in \mathcal{A}$, so $A=A^{\prime}$ by the minimality of $A$. Therefore, $\mathcal{P}:=A$ satisfies the properties required in the lemma.

Proposition 3.2. If $\mathcal{P}=M$, then the foliation fibers over the circle.

Proof. This is immediate from Theorem 2.3 and the easy fact that $\mathcal{F}$ is, in this case, without holonomy.

The connected components of $M-\mathcal{P}$ will be called the gaps of $\mathcal{P}$.

Lemma 3.3. Suppose that $\mathcal{P}$ is a proper subset of $M$, and the $\Gamma$-action is not fixing. Then

1) each gap is bounded by two leaves of $\mathcal{P}$, and

2) there exists a finite open cover $\left\{V_{1}, \ldots, V_{k}\right\}$ of $M$ by foliated products such that each $V_{i}$ is bounded by two leaves in $\mathcal{P}$.

Proof. We first remark that each connected component of $W=M-\mathcal{P}$ is a foliated product. In fact, by $[3,5.2 .9]$, only finitely many connected components of $W$ fail to be foliated products. Suppose that there are connected components of $W$ which are not foliated products and denote them by $W_{1}, \ldots, W_{l}$. Since any homeomorphism $\gamma \in \Gamma$ must send each $W_{i}$ into some (possibly the same) $W_{j}$, one obtains a homomorphism of $\Gamma$ into the group of permutations of $l$ symbols, from which it follows that some finiteindex subgroup of $\Gamma$ sends each $W_{i}$ to itself. In particular, this subgroup would permute the boundary components of a $W_{i}$. By $[3,5.2 .5]$ the boundary of a connected $\mathcal{F}$-saturated open set consists of the union of a finite number of leaves, which in this case must be elements of $\mathcal{P}$. But then a finite-index subgroup of $\Gamma$ would send one leaf in $\mathcal{P}$ to itself, contradicting the assumption that the action is not fixing.

A leaf in $\mathcal{P}$ will be called a border leaf of $\mathcal{P}$ if it is a component of the boundary of a gap. If $\mathcal{P}$ is not all of $M$, there must be a countable infinity of gaps, since otherwise $\mathcal{P}$ would be contained in the union of the finitely many boundary leaves of a finite number of connected $\mathcal{F}$-saturated open sets. Each border leaf $L$ of $\mathcal{P}$ is a boundary component of a gap and on the side of $L$ opposite the gap a sequence of leaves in $\mathcal{P}$ accumulates on $L$.

If a leaf $L$ of $\mathcal{P}$ does not bound a gap, then $L$ is a limit of sequences of leaves in $\mathcal{P}$ on both of its sides, so that $[\mathbf{3}, 5.3 .4]$ (due to Dippolito [5]) immediately yields a foliated product neighborhood of $L$.

We claim that boundary of each gap of $\mathcal{P}$ consists of two (distinct) leaves in $\mathcal{P}$, and that the closure of each gap is contained in a foliated product bounded by two leaves in $\mathcal{P}$. The interiors of these foliated products together with the interiors of the foliated products of the previous paragraph form an open cover for $M$. Since $M$ is compact, we can extract a finite open cover. 
All that is left is to prove the claim. Let $W$ denote a gap of $\mathcal{P}$. We have seen that it is a foliated product. Denote by $B$ the base manifold. The boundary of $W$ consists of two (distinct) leaves in $\mathcal{P}$. (There are not more than two leaves by Theorem 2.1. If a single leaf of $\mathcal{P}$ bounded $W$ on both sides, this would be an isolated leaf, which is not the case.) The boundary leaves are homeomorphic to $B$ (notice that the boundary leaves of $\widehat{W}$ are homeomorphic to the base of the fibration on $\widehat{W}$ ) and $\widehat{W}$ maps bijectively onto the closure of $W$ under the map $\hat{\imath}$. Since the boundary leaves $L_{1}$ and $L_{2}$ are compact, it is possible to find neighborhoods $U_{1}$ and $U_{2}$ of $L_{1}$ and $L_{2}$, respectively, such that $\left.U_{i}\right|_{\mathcal{L}}$ is a trivial bundle over $L_{i}, i=1,2$, not necessarily $\mathcal{F}$-saturated. To obtain $\mathcal{F}$-saturated $U_{i}$, one applies $[\mathbf{3}, 5.3 .4]$. The union of $W, U_{1}$ and $U_{2}$ (for sufficiently small $U_{i}$ ) gives the desired neighborhood.

For $x \in \mathcal{P}$, let $L_{x}$ be the leaf of $\mathcal{F}$ that contains $x$. Define an equivalence relation $\sim$ on $\mathcal{P}$ by specifying that $x \sim y$ if either $L_{x} \cup L_{y}$ is the boundary of a gap of $\mathcal{P}$, or $L_{x}=L_{y}$. (In particular, if $\mathcal{P}=M$, then $x \sim y$ if and only if $L_{x}=L_{y}$.) Note that each equivalence class is either a leaf or the union of two leaves.

Lemma 3.4. If $\Gamma$ is as in Theorem 1.1 and $\mathcal{F}$ has a compact leaf, but the $\Gamma$-action is not fixing, then $\mathcal{P} / \sim$ is homeomorphic to $S^{1}$.

Proof. We may assume $\mathcal{P}$ is a proper subset of $M$; otherwise, the desired conclusion follows from Proposition 3.2. It is immediate from Lemma 3.3 (and the "waterfall construction" described in $[3,3.3 .7]$ ) that there exists a closed transversal, $\alpha$, of $\mathcal{F}$ that intersects each leaf of $\mathcal{P}$ exactly once. The intersection $\alpha \cap \mathcal{P}$ is a perfect set in the embedded circle $\alpha$, and the saturations of the gaps of this perfect set are the gaps of $\mathcal{P}$. Thus, the desired conclusion follows from the elementary observation that, by identifying the two endpoints of each of the gaps of $\alpha \cap \mathcal{P}$ to a single point, we obtain a quotient that is homeomorphic to a circle.

Suppose the $\Gamma$-action is not fixing, and let $\mathcal{P}$ be as in Lemma 3.1. The action of $\Gamma$ on $\mathcal{P}$ factors through to an action of $\Gamma$ by homeomorphisms of $\mathcal{P} / \sim$. Now Lemma 3.4 implies that $\mathcal{P} / \sim$ is homeomorphic to $S^{1}$, so, by assumption, $\Gamma$ must have a finite orbit on $\mathcal{P} / \sim$. This finite orbit yields a $\Gamma$-invariant, finite collection of compact leaves in $\mathcal{P}$. Then some finite-index subgroup of $\Gamma$ fixes each of these compact leaves. This proves Theorem 1.1.

\section{Proof of Corollary 1.2.}

Suppose that $\mathcal{F}$ admits a transverse invariant measure.

If $\mathcal{F}$ has a compact leaf, then Theorem 1.1 implies that some finite-index subgroup $\Gamma^{\prime}$ of $\Gamma$ fixes some compact leaf $L$. Then $\Gamma^{\prime}$ fixes the atomic 
measure $\mu$ supported on the single leaf $L$, so the conclusion of Corollary 1.2 holds.

Thus, we may assume that no leaf of $\mathcal{F}$ is compact. Therefore, from Theorems 2.2 and 2.3, it follows that $\mathcal{F}$ is a minimal foliation (that is, every leaf is dense in $M$ ). Then Corollary 4.2 below completes the proof of Corollary 1.2.

Corollary 4.2 is stated in greater generality than needed for the proof of Corollary 1.2 because it will be used in the given form for the proof of Theorem 1.3.

The next observation follows from the proof of [10, Thm. X.2.3.3, p. 272].

Proposition 4.1. Let $U$ be a connected, $\Gamma$-invariant, $\mathcal{F}$-saturated open set. We suppose that the boundary of $U$ is either empty or consists of finitely many $\Gamma$-invariant compact leaves. We also suppose that each leaf of $\mathcal{F}$ in $U$ is dense in $U$. Let $\mu$ be a bounded transverse invariant measure on $U$.

If $\mu^{\prime}$ is another bounded transverse invariant measure on $U$, then $\mu^{\prime}$ is a scalar multiple of $\mu$.

Corollary 4.2. Let $U$ be a connected, $\Gamma$-invariant, $\mathcal{F}$-saturated open set. We suppose that the boundary of $U$ is either empty or consists of finitely many $\Gamma$-invariant compact leaves. We also suppose that each leaf of $\mathcal{F}$ in $U$ is dense in $U$. Let $\mu$ be a bounded transverse invariant measure on $U$. Then

1) $\mu$ is $\Gamma$-invariant; and

2) $[\Gamma, \Gamma]$ is a finite-index subgroup of $\Gamma$ that fixes each leaf in $U$.

Proof. (1) For each $\gamma \in \Gamma$, Proposition 4.1 implies there is some $c(\gamma) \in \mathbb{R}^{+}$, such that $\gamma_{*} \mu=c(\gamma) \mu$. It is easy to see that $c: \Gamma \rightarrow \mathbb{R}^{+}$is a homomorphism, so, because $\mathbb{R}^{+}$is abelian and has no nontrivial finite subgroups, Lemma 4.3 implies $c(\Gamma)=1$. Thus, $\mu$ is $\Gamma$-invariant.

(2) We use the notations of Theorem 2.4, where the Sacksteder flow $\Phi_{t}$ is defined. Integration of $\mu$ over closed curves representing elements of $\pi_{1}(U)$ yields a homomorphism $\rho: \pi_{1}(U) \rightarrow \mathbb{R}$ whose image group, $P(\mu)$, is called the group of periods of $\mu[\mathbf{3}, 9.3 .4]$. This is a finitely generated abelian subgroup of $\mathbb{R}$. The group of periods can be characterized by the following property $([\mathbf{3}, 9.3 .6]): \Phi_{t}$ sends every leaf to itself exactly when $t \in P(\mu)$.

Define for each $p \in U$ and each $\gamma \in \Gamma$ a class $[t] \in \mathbb{R} / P(\mu)$ where $t$ is any real number such that $\Phi_{t}(p)$ lies in the leaf of $\gamma(p)$. Then the correspondence $\gamma \mapsto[t]$ gives a well-defined homomorphism, $h$ from $\Gamma$ into $\mathbb{R} / P(\mu)$. Because $\mathbb{R} / P(\mu)$ is abelian, we know that $[\Gamma, \Gamma]$ is in the kernel of $h$, so $[\Gamma, \Gamma]$ sends every leaf to itself. Furthermore, Lemma 4.3 below asserts that $[\Gamma, \Gamma]$ is a finite-index subgroup of $\Gamma$.

The following well-known observation is easy to prove. 
Lemma 4.3. If each homomorphism of a finitely generated group $\Gamma$ into the group of homeomorphisms of the circle has a finite orbit, then $A:=\Gamma /[\Gamma, \Gamma]$ is a finite group.

\section{Proof of Theorem 1.3.}

The following lemma is a slight generalization of the Thurston Stability Theorem [15]:

- We only assume the action of $\Gamma$ is germinal, rather than being globally defined; and

- we do not assume that each element of $\Gamma$ is defined in a neighborhood of 0 , but only on a set $X$ that accumulates at 0 .

Thurston's original proof (and many others, such as [11]) can easily be generalized to this setting.

Lemma 5.1 (Thurston, cf. [15]). Suppose $\Gamma$ is a finitely generated group, $X$ is a compact subset of $[0,1]$ that accumulates at 0 , and, for each $\gamma \in \Gamma$, we have a $C^{1}$ diffeomorphism $\phi_{\gamma}:\left[0, a_{\gamma}\right) \rightarrow\left[0, b_{\gamma}\right)$, for positive constants $a_{\gamma}$ and $b_{\gamma}$. Assume

1) $\Gamma /[\Gamma, \Gamma]$ is finite; and

2) for each $\gamma_{1}, \gamma_{2} \in \Gamma$, there exists $c>0$, such that $\left.\phi_{\gamma_{1} \gamma_{2}}\right|_{[0, c] \cap X}=$ $\left.\phi_{\gamma_{1}} \phi_{\gamma_{2}}\right|_{[0, c] \cap X}$.

Then there exists $a>0$, such that, for every $\gamma \in \Gamma$ and $x \in[0, a) \cap X$, we have $\phi_{\gamma}(x)=x$.

All the assumptions of Theorem 1.3 are in force from now on.

Lemma 5.2. Let $\Gamma^{\prime}$ be a finite-index subgroup of $\Gamma$ and suppose that $W$ is a connected, open, $\Gamma^{\prime}$-invariant, $\mathcal{F}$-saturated subset of $M$ whose boundary components are $\Gamma^{\prime}$-invariant compact leaves. If the compact leaves accumulate on a boundary component $L$ of $\widehat{W}$, then all the compact leaves in some foliated neighborhood of $L$ in $\widehat{W}$ are also $\Gamma^{\prime}$-invariant.

Proof. Let $U$ be a connected, $\widehat{\mathcal{F}}$-saturated neighborhood of $L$ in $\widehat{W}$. We may assume $U$ is so small that there is a complete $C^{1}$ transversal $\alpha:[0,1] \rightarrow U$ of $U$, such that each compact leaf in $U$ meets $\alpha$ exactly once. We may assume $\alpha(0) \in L$.

As the $\Gamma$-action and the foliation (hence the local holonomy maps) are $C^{1}$, we can construct, for each $\gamma \in \Gamma^{\prime}$, a $C^{1}$ diffeomorphism $\phi_{\gamma}:\left[0, a_{1}\right] \rightarrow\left[0, a_{2}\right]$, for positive constants $a_{i}$, such that $\alpha\left(\phi_{\gamma}(t)\right)$ is on the same leaf as $\gamma(\alpha(t))$, for each $t \in\left[0, a_{1}\right]$. Let

$$
X=\{x \in[0,1] \mid \alpha(x) \text { is on a compact leaf }\} .
$$

For each $\gamma_{1}, \gamma_{2} \in \Gamma$, there exists $c>0$, such that $\alpha\left(\phi_{\gamma_{1} \gamma_{2}}(t)\right)$ is on the same leaf as $\alpha\left(\phi_{\gamma_{1}}\left(\phi_{\gamma_{2}}(t)\right)\right)$, for each $t \in[0, c)$. Then, for $x \in X \cap[0, c)$, 
we must have $\phi_{\gamma_{1} \gamma_{2}}(x)=\phi_{\gamma_{1}}\left(\phi_{\gamma_{2}}(x)\right)$. Also, from Lemma 4.3, we know that $\Gamma^{\prime} /\left[\Gamma^{\prime}, \Gamma^{\prime}\right]$ is finite. Therefore, Lemma 5.1 applies, so we conclude that there is an interval $[0, a)$, such that $\alpha(x)$ is on the same leaf as $\gamma(\alpha(x))$, for all $x \in X \cap[0, a)$. So $\Gamma^{\prime}$ fixes the compact leaf containing $\alpha(x)$, for each $x \in X \cap[0, a)$.

Lemma 5.3. If a finite group $H$ acts on the circle by orientation preserving homeomorphisms, then the action of the commutator subgroup $[H, H]$ is trivial.

Proof. By a standard averaging procedure $H$ can be assumed to act by isometries of a metric on the circle. Therefore the action is conjugate to a homomorphism of $H$ into the group of rotations of the circle, which is an abelian group. It follows that the commutator subgroup must act trivially.

Lemma 5.4. Let $\Gamma^{\prime}$ be a finite-index subgroup of $\Gamma$ and suppose that $W \subset$ $M$ is a connected, open, $\Gamma^{\prime}$-invariant, $\mathcal{F}$-saturated subset, such that the boundary components of $W$ are $\Gamma^{\prime}$-invariant compact leaves, and every compact leaf in $W$ is $\Gamma^{\prime}$-invariant. Then all leaves in $W$ are $\left[\Gamma^{\prime}, \Gamma^{\prime}\right]$-invariant.

Proof. From Theorem 2.5 and the hypothesis about $W$ it follows that the complement in $W$ of the set of compact leaves is a countable union of connected, $\Gamma^{\prime}$-invariant, $\mathcal{F}$-saturated sets $U_{i}, i=1,2, \ldots$, such that the boundary of each $U_{i}$ is the union of at most 2 compact, $\Gamma^{\prime}$-invariant leaves. Since there are no compact leaves in each of the $U_{i}$, each leaf of $\left.\mathcal{F}\right|_{U_{i}}$ is, by assumption, without holonomy. Theorem 2.3 implies that either

1) $\left.\mathcal{F}\right|_{U_{i}}$ fibers over the circle; or

2) $\left(\widehat{U}_{i}, \widehat{\mathcal{F}}\right)$ fibers over $[0,1]$; or

3) every leaf of $\left.\mathcal{F}\right|_{U_{i}}$ is dense in $U_{i}$.

In Case 1 , we obtain a $C^{1}$ action of $\Gamma^{\prime}$ on the circle. This action has to be finite, that is, a finite-index subgroup of $\Gamma^{\prime}$ must leave invariant each leaf in $U_{i}$. From Lemma 5.3, we know that this normal subgroup contains the commutator subgroup $\left[\Gamma^{\prime}, \Gamma^{\prime}\right]$.

In Case 2, we obtain a $C^{1}$ action of $\Gamma^{\prime}$ on $[0,1]$. Lemma 5.1 implies that this action is trivial.

In Case 3, we can apply Theorem 2.4(1) and Corollary 4.2(2) to conclude that each leaf in $U_{i}$ is $\Gamma^{\prime}$-invariant.

Therefore, $\left[\Gamma^{\prime}, \Gamma^{\prime}\right]$ fixes every leaf of $W$.

We now conclude the proof of Theorem 1.3. It suffices to show that there is a finite-index subgroup $\Gamma^{\prime}$ of $\Gamma$, such that $\Gamma^{\prime}$ fixes every compact leaf, for then Lemma 5.4 shows that $\left[\Gamma^{\prime}, \Gamma^{\prime}\right]$ fixes every leaf (and Lemma 4.3 implies that $\left[\Gamma^{\prime}, \Gamma^{\prime}\right]$ has finite index in $\Gamma$ ). 
We may assume that $\mathcal{F}$ has a compact leaf. Then Theorem 1.1 states that there is a finite-index subgroup $\Gamma^{\prime}$ of $\Gamma$, and a compact leaf $L_{0}$ of $\mathcal{F}$, such that $\Gamma^{\prime}$ fixes $L_{0}$. Let $W_{0}$ be the complement of the union of all the compact leaves of $\mathcal{F}$. From Theorem 2.1, we know that only finitely many components $U_{1}, U_{2}, \ldots, U_{k}$ of $W_{0}$ fail to be foliated products, and that each of these components has only finitely many boundary leaves, so, replacing $\Gamma^{\prime}$ with a finite-index subgroup, we may assume that

$\Gamma^{\prime}$ fixes each $U_{i}$, and its boundary components.

To complete the proof, we will show that $\Gamma^{\prime}$ fixes every compact leaf. Let $W$ be a component of the complement of the union of the $\Gamma^{\prime}$-invariant compact leaves of $\mathcal{F}$. It suffices to show that every compact leaf in $W$ is $\Gamma^{\prime}$-invariant.

Let $L$ be any leaf on the boundary of $W$. By definition of $W$, we know that $L$ is $\Gamma^{\prime}$-invariant.

If the compact leaves in $W$ accumulate on $L$, then Lemma 5.2 implies that every compact leaf in $W$ is $\Gamma^{\prime}$-invariant.

If the compact leaves in $W$ do not accumulate on $L$, then there is a component $U$ of $W_{0}$ such that $L$ is part of the boundary of $U$, and $U$ is contained in $W$. We will show that $U=W$, so there are no compact leaves in $W$. If $U$ is not a foliated product, then from (5.5), we know that $\Gamma^{\prime}$ fixes each boundary component of $U$, so $U=W$ as desired. Now suppose that $U$ is a foliated product. Then the boundary of $U$ consists of at most two compact leaves. One of these boundary leaves is $L$, which is known to be $\Gamma^{\prime}$-invariant. Thus the second boundary leaf (if it exists) must also be $\Gamma^{\prime}$-invariant, so $U=W$, as desired.

Acknowledgement. The authors would like to thank the Isaac Newton Institute for Mathematical Sciences (Cambridge, U.K.) for providing a congenial environment to carry out this research; and S. Hurder and L. Conlon, for a number of helpful discussions and guidance in the literature of codimension-one foliations. D.W. was partially supported by a grant from the National Science Foundation (DMS-9801136).

\section{References}

[1] M. Burger and N. Monod, Bounded cohomology of lattices in higher rank Lie groups, J. Eur. Math. Soc., 1(2) (1999), 199-235; erratum 1, 3 (1999), 338, MR 2000g:57058a, MR 2000g:57058b, Zbl 0932.22008.

[2] _ Continuous bounded cohomology and applications, preprint.

[3] A. Candel and L. Conlon, Foliations I, Graduate Studies in Mathematics, 23, American Mathematical Society, 2000, CMP 1732 868, Zbl 0936.57001.

[4] J. Cantwell and L. Conlon, Tischler fibrations of open, foliated sets, Ann. Inst. Fourier (Grenoble), 31(2) (1981), vi, 113-135, MR 83e:57021, Zbl 0442.57007. 
[5] P. Dippolito, Codimension one foliations of closed manifolds, Ann. of Math., 107 (1978), 403-453, MR 84e:57025, Zbl 0418.57012.

[6] É. Ghys, Actions de réseaux sur le cercle, Invent. Math., 137 (1999), 199-231, MR 2000j:22014.

[7] C. Godbillon, Feuilletages, Birkhäuser, Boston, 1991, MR 93i:57038, Zbl 0724.58002.

[8] A. Haefliger, Variétés feuilletées, Ann. Scuola Norm. Sup. Pisa, 16 (1962), 367-397, MR 32 \#6487, Zbl 0122.40702.

[9] G. Hector, Sur les feuilletages presque sans holonomie, C. R. Acad. Sci. Paris, 274 (1972), 1703-1706, MR 46 \#2687, Zbl 0233.57012.

[10] G. Hector and U. Hirsch, Introduction to the Geometric Theory of Foliations, part B, Aspects of Mathematics, 1983, Vieweg, MR 92f:57037, Zbl 0552.57001.

[11] R. Lutz and M. Goze, eds., Nonstandard analysis, Lecture Notes in Mathematics, 881, Springer, 1981, MR 83i:03103, Zbl 0506.03021.

[12] T. Mizutani, S. Morita and T. Tsuboi, The Godbillon-Vey classes of codimension one foliations which are almost without holonomy, Ann. Math., 113 (1981), 515-527, MR 83m:57020, Zbl 0465.57010.

[13] G. Reeb and P. Schweitzer, Un théoreme de Thurston établi au moyen de l'analyse non standard, in 'Differential Topology, Foliations and Gelfand-Fuks Cohomology,' P.A. Schweitzer, ed., (Proc. Sympos., Pontifícia Univ. Católica, Rio de Janeiro, January 1976), Lecture Notes in Math., 652, Springer, New York, (1978), 138, MR 58 \#18491b, Zbl 0405.58011.

[14] R. Sacksteder, Foliations and pseudogroups, Amer. J. Math., (1965), 79-102, MR 30 \#4268, Zbl 0136.20903.

[15] W. Thurston, A generalization of the Reeb stability theorem, Topology, 13 (1974) 347-352, MR 50 \#8558, Zbl 0305.57025.

Received August 17, 2000 and revised March 17, 2001.

Department of Mathematics (CB 1146)

WASHINGTON UNIVERSITY

ST. Louis, MO 63130

E-mail address: feres@math.wust1.edu

Department of Mathematics

OKlahoma State University

STILLWATER, OK 74078

E-mail address: dwitte@math.okstate.edu 